\title{
Comprehensive characterization of erythroid- specific enhancers in the genomic regions of human Krüppel-like factors
}

\author{
Qian Xiong ${ }^{1,2+}$, Zhaojun Zhang ${ }^{1 \dagger}$, Kai-Hsin Chang ${ }^{3 \dagger}$, Hongzhu Qu ${ }^{1,4}$, Hai Wang ${ }^{1,2,3}$, Heyuan Qi ${ }^{1,2}$, Yajuan Li ${ }^{1}$, \\ Xiuyan Ruan ${ }^{1}$, Yaran Yang ${ }^{1}$, Yadong Yang ${ }^{1}$, Yanming $\mathrm{Li}^{1,2}$, Richard Sandstrom ${ }^{4}$, Peter J Sabo ${ }^{4}$, Qiliang Li ${ }^{5}$, \\ George Stamatoyannopoulos ${ }^{5}$, John A Stamatoyannopoulos ${ }^{4^{*}}$ and Xiangdong Fang ${ }^{1,5^{*}}$
}

\begin{abstract}
Background: Mapping of DNase I hypersensitive sites (DHSs) is a powerful tool to experimentally identify cisregulatory elements (CREs). Among CREs, enhancers are abundant and predominantly act in driving cell-specific gene expression. Krüppel-like factors (KLFs) are a family of eukaryotic transcription factors. Several KLFs have been demonstrated to play important roles in hematopoiesis. However, transcriptional regulation of KLFs via CREs, particularly enhancers, in erythroid cells has been poorly understood.

Results: In this study, 23 erythroid-specific or putative erythroid-specific DHSs were identified by DNase-seq in the genomic regions of 17 human KLFs, and their enhancer activities were evaluated using dual-luciferase reporter (DLR) assay. Of the 23 erythroid-specific DHSs, the enhancer activities of 15 DHSs were comparable to that of the classical enhancer HS2 in driving minimal promoter (minP). Fifteen DHSs, some overlapping those that increased minP activities, acted as enhancers when driving the corresponding KLF promoters (KLF-Ps) in erythroid cells; of these, $10 \mathrm{DHSs}$ were finally characterized as erythroid-specific KLF enhancers. These 10 erythroid-specific KLF enhancers were further confirmed using chromatin immunoprecipitation coupled to sequencing (ChIP-seq) data-based bioinformatic and biochemical analyses.

Conclusion: Our present findings provide a feasible strategy to extensively identify gene- and cell-specific enhancers from DHSs obtained by high-throughput sequencing, which will help reveal the transcriptional regulation and biological functions of genes in some specific cells.
\end{abstract}

Keywords: DHS profiling, High-throughput sequencing, Erythroid, Enhancer, Krüppel-like factors

\section{Background}

Biological processes such as proliferation, apoptosis, differentiation, development, and aging require elaborately orchestrated spatial and temporal gene expression, which are often under the control of cis-regulatory elements (CREs). CREs, including promoters, enhancers, silencers, insulators and locus control regions (LCRs) etc., are abundant in the human genome [1]. Characterization of

\footnotetext{
* Correspondence: jstam@u.washington.edu; fangxd@big.ac.cn

†Equal contributors

${ }^{4}$ Department of Genome Sciences, University of Washington, Seattle, WA 98195, USA

${ }^{1}$ CAS Key Laboratory of Genome Sciences and Information, Beijing Institute of Genomics, Chinese Academy of Sciences, Beijing 100101, P.R. China Full list of author information is available at the end of the article
}

CREs in the genome contributes to understand the complexities of gene transcription and expression in different biological systems [2,3]. In the past decade, the project ENCyclopedia of DNA Elements (ENCODE) has facilitated the prediction of functional elements including CREs in the human genome [4]. However, CRE characterization in terms of gene and cell specificities as well as chromatin context dependency remains a huge challenge.

Nucleosome-depleted DNA regions, characterized by their sensitivity to nuclease digestion, are closely associated with almost all known classes of active CREs. In contrast, DNA regions tightly wrapped in nucleosomes and higherorder structures are more resistant to nuclease digestion. Therefore, DNase I hypersensitive sites (DHSs) mark many 
types of CREs [2,5]. Individual DHSs within small regions of the genome (10-20 kb) have been traditionally identified using Southern blot analysis [6]. However, this laborintensive and low-throughput approach cannot be scaled to study large chromosomal regions and entire genomes. By hybridizing DNase I-digested fragments to tiled microarrays, the DNase-chip assay provides an undirected, unbiased, highly sensitive and specific strategy to simultaneously identify thousands of DHSs within any region of interest or even the entire genome, with a resolution of 200-500 bases [7]. Furthermore, DNase-seq (identification of DNase I-digested fragments by next-generation sequencing) allows genome-wide mapping of DHSs with base-pair resolution [2]. Using these high-throughput technologies, DHS mapping is emerging as a powerful tool for locating open chromatin regions that encompassing many types of CREs within the genome $[2,3,8]$ and thus it facilitates the delineation of the roles of DHSs in regulating tissue- and developmental stage-specific expression of nearby genes $[9,10]$.

Enhancers are the most variable CREs that can regulate the expression of genes from a long distance and in a position- and orientation-independent manner [11]. In general, it is accepted that enhancers function by first recruiting sequence-specific transcription factors (TFs) that recognize short DNA motifs within the enhancers. Upon binding to enhancers, the sequence-specific TFs recruit mediator complexes, histone modifiers and chromatin remodelers to activate the transcription of target genes [12]. Enhancers often exist in a cell- and developmental stage-specific manner [13], and the distribution of cell-specific enhancers correlates well with cellspecific gene expression [14], suggesting that they are the primary force driving spatial- or temporal-specific gene expression. To date, several lines of evidences have demonstrated the roles of erythroid-specific enhancers in driving erythroid-specific gene expression. LCR at the $\beta$-globin locus is the most prominent erythroid enhancer that exerts a strong effect specifically on erythroid cells. This LCR enhances the developmental stage-specific expression of globin genes and the expression of linked heterogeneous non-globin genes in erythroid cells by interacting with respective promoters [15]. HS2, a classical enhancer located in LCR, appears to be functional in erythroid cells at both embryonic and adult developmental stages, suggesting its crucial roles in the activation of globin genes in erythroid cells throughout ontogenesis [16]. Other erythroid-specific enhancers have also been found in the genomic regions of GATA1, stem cell leukemia $(S C L)$, L-type pyruvate kinase and 5-aminolevulinate synthase 2 (ALAS2) genes [17-20], which may contribute to the restricted expression of these genes in the erythroid lineage. A strikingly large number of enhancers have been systematically identified in erythroid K562 cells using chromatin immunoprecipitation followed by genome tiling array (ChIP-chip) analysis [14]. The characterization of these and other erythroid or erythroid-specific enhancers in the human genome will facilitate the understanding of regulation and functions of associated genes in erythroid cells.

Krüppel-like factors (KLFs) are a subfamily of zincfinger proteins that contain three tandem $\mathrm{Cys}_{2} \mathrm{His}_{2}$ zinc fingers at the highly conserved carboxyl terminus. KLFs are important components of the eukaryotic cellular transcriptional machinery. By regulating the expression of several genes driven by GC-rich or CACCCcontaining promoters, KLFs participate in many biological processes, including hematopoiesis, adipogenesis, stem cell maintenance, and tumorigenesis [21]. In particular, several KLFs have been demonstrated to play crucial roles in erythroid differentiation. Globin genes, including $\alpha-, \varepsilon-, \gamma$ - and $\beta$-globin genes, are prominent biomarkers in erythroid cells, and their spatial and temporal expression is closely correlated with erythroid differentiation and development [22]. KLF1 (EKLF), an erythroid-specific TF, activates adult $\beta$-globin gene expression [23] and regulates gene switching from $\gamma$ - to $\beta$-globin [24] as well as definitive hematopoiesis. Other KLFs mainly play significant roles in primitive hematopoiesis. For example, KLF4 activates the expression of $\alpha$ - and $\gamma$-globin genes $[25,26]$. Expression of embryonic $\varepsilon$ - and fetal $\gamma$-globin genes is stimulated by KLF2 [27], KLF11 [28,29], and KLF13 [30] but is suppressed by KLF3 [31] and KLF8 [32]. In addition, KLF6 [33] and KLF17 [34] are required for primitive hematopoiesis. Hematopoietic defects or anemia have been observed in several Klf-knockout mice, including mice lacing Klf1 [35], Klf2 [27], Klf3 [36], Klf6 [33], and Klf13 [37]. Interestingly, cross-regulation among KLFs has been reported during erythropoiesis, erythroid differentiation, and globin gene regulation [36,38,39]. However, till date, few studies have been conducted to investigate the cis-transcriptional regulation of KLFs by erythroid-specific enhancers, with the exception of the study on murine KLF1 enhancers [40]. Therefore, characterization of erythroid-specific enhancers will shed light on molecular mechanisms that regulate transcription, expression, and functions of KLFs in erythroid cells.

Here, we characterized gene- and cell-specific enhancers in the genomic regions of human KLFs extensively by combining high-throughput sequencing data as well as biochemical and bioinformatic analyses. Our mRNA-seq data in human embryonic stem cells (HESC) and three primary erythroid cell types demonstrated that human KLFs, including KLF1, KLF3, KLF6, KLF9, KLF10, KLF11, KLF13, and KLF16, were up-regulated in erythroid cells as compared to HESC. We also mapped DHSs in the genomic regions of 17 human KLFs across 
four erythroid and seven non-erythroid cell types and screened out 23 erythroid-specific or putative erythroidspecific DHSs. Using the dual-luciferase reporter (DLR) assay, we identified 10 (43\%) erythroid-specific enhancers embedded in the genomic regions of KLF1, KLF6, KLF9, and KLF13. The nature of these identified erythroidspecific enhancers was confirmed by a series of bioinformatic and biochemical analyses, contributing to understand the mechanism by which KLFs are regulated in erythroid cells. Our present findings provide a feasible strategy to characterize cell- and gene-specific enhancers from DHSs generated from high-throughput sequencing across various cell types, and to facilitate the illustration of transcriptional regulation and functions of genes in specific cell types.

\section{Results}

Expression of some KLFs is up-regulated in erythroid cells Expression patterns of KLFs were obtained from the mRNA-seq dataset that was originally designed to explore the dynamic transcriptomes during human erythroid differentiation and development (Yang Y, Wang H, Chang KH, Qu H, Zhang Z, Xiong Q, Qi H, Cui P, Lin Q, Ruan X, et al: Transcriptome dynamics during human erythroid differentiation and development, submitted). The following cell types were examined: undifferentiated HESC, embryonic stem cells-derived erythroid cells (ESER), fetal liver-derived erythroid cells (FLER), and adult mobilized peripheral blood CD34+ cells-derived erythroid cells (PBER). As shown in Figure 1A, of the 17 KLFs examined, the

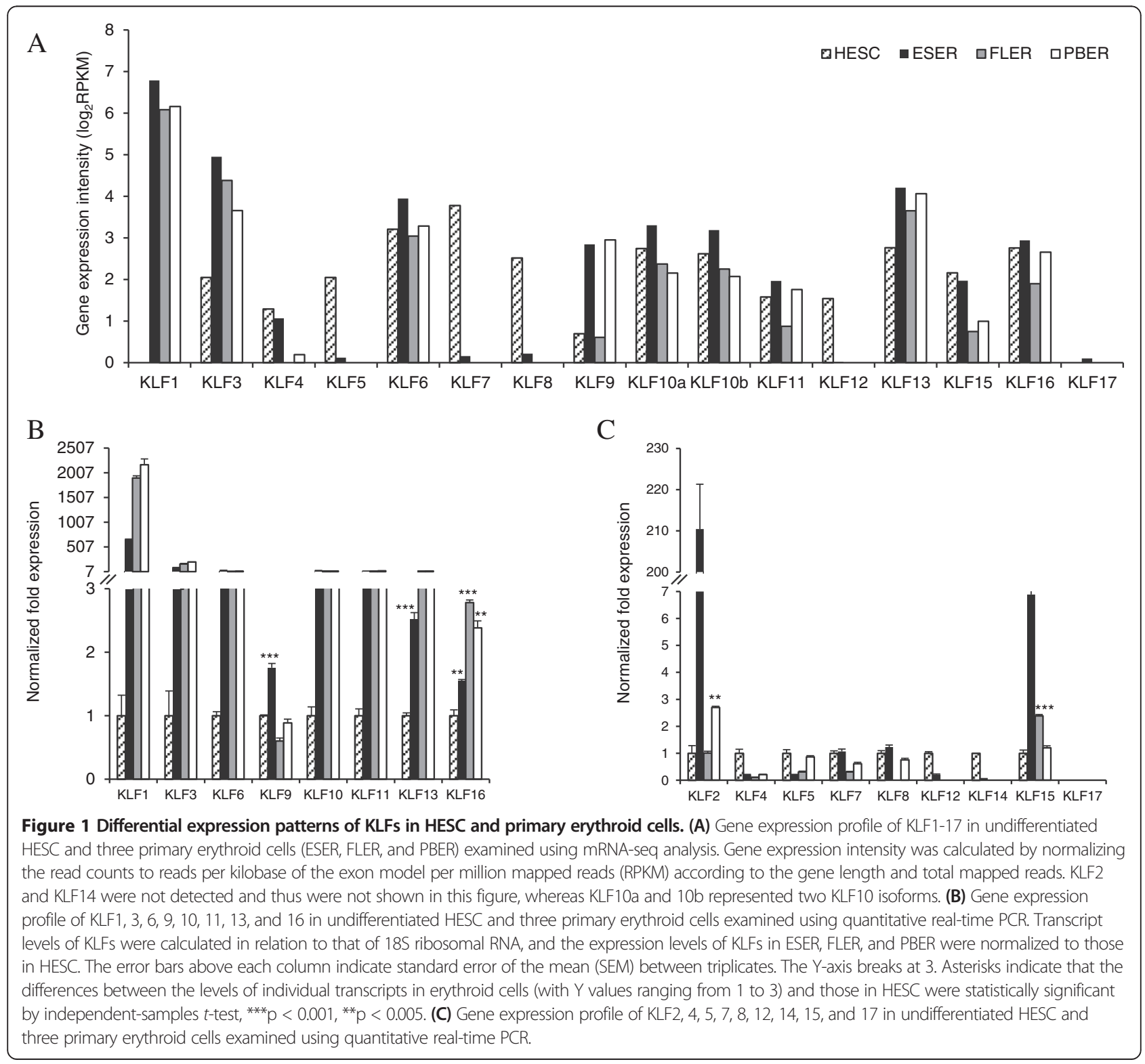


expressions of KLF2 and KLF14 were not detected, whereas KLF17 was poorly expressed in ESER. The expressions of KLF4, KLF5, KLF7, KLF8, KLF12, and KLF15 in HESC were higher than those in erythroid cells, whereas those of the remaining eight KLFs were higher in erythroid cells than in HESC. The expressions of all the 17 KLFs were further evaluated using quantitative real-time PCR. With the exception of KLF2 and KLF15, the expression patterns of the remaining 15 KLFs measured by PCR correlated well with mRNA-seq results (Figure $1 \mathrm{~B}, \mathrm{C}$ ). The cause of the inconsistency in KLF2 and KLF15 measurement is currently not clear but it could be platform related. Nevertheless, we proposed that the higher expressions of the eight KLFs detected by both two platforms may be attributed to the presence of erythroid-specific enhancers.

\section{DHSs in KLF genomic regions are distributed diversely in various cell types}

The DNase-seq dataset used in this study was generated by the University of Washington [41]. The dataset is composed of DNase-seq data in four erythroid cell types, including three primary erythroid cells (ESER, FLER, and PBER) and erythroleukemia K562 cells, to cover all the possible DHSs in the erythroid lineage and seven non-erythroid cell types, including HESC, GM12878, hTH2, HeLa, HepG2, CACO2, and BJ, to differentiate erythroid-specific DHSs from non-erythroid ones. A false discovery rate (FDR) threshold of $0.5 \%$ was used to define DHSs in each cell type. DHS mapping was profiled for all the $K L F$ gene loci from $70 \mathrm{~kb}$ upstream of the transcription start sites (TSSs) to $20 \mathrm{~kb}$ downstream of the poly (A) sites. The current coverage of gene loci was determined based on the following reasons: First, the regions covering approximately $100 \mathrm{~kb}$ encompassed almost all intensive DHSs around the corresponding KLFs in the four cell types studied (Figure 2, Additional file 1: Figures S1 and S2). Second, CTCF binding is reported to mark boundary elements between neighboring genes [42]. These approximate $100-\mathrm{kb}$ regions contain such ubiquitous CTCF-binding sites in the various cell types employed in the present study (data from UCSC Browser). The diverse distribution patterns of KLF DHSs among the various established cell types and HESC were shown in Figure 2, Additional file 1: Figures S1 and S2, coinciding with the varying expression levels of $K L F$ genes in these cell types. DHSs were considered

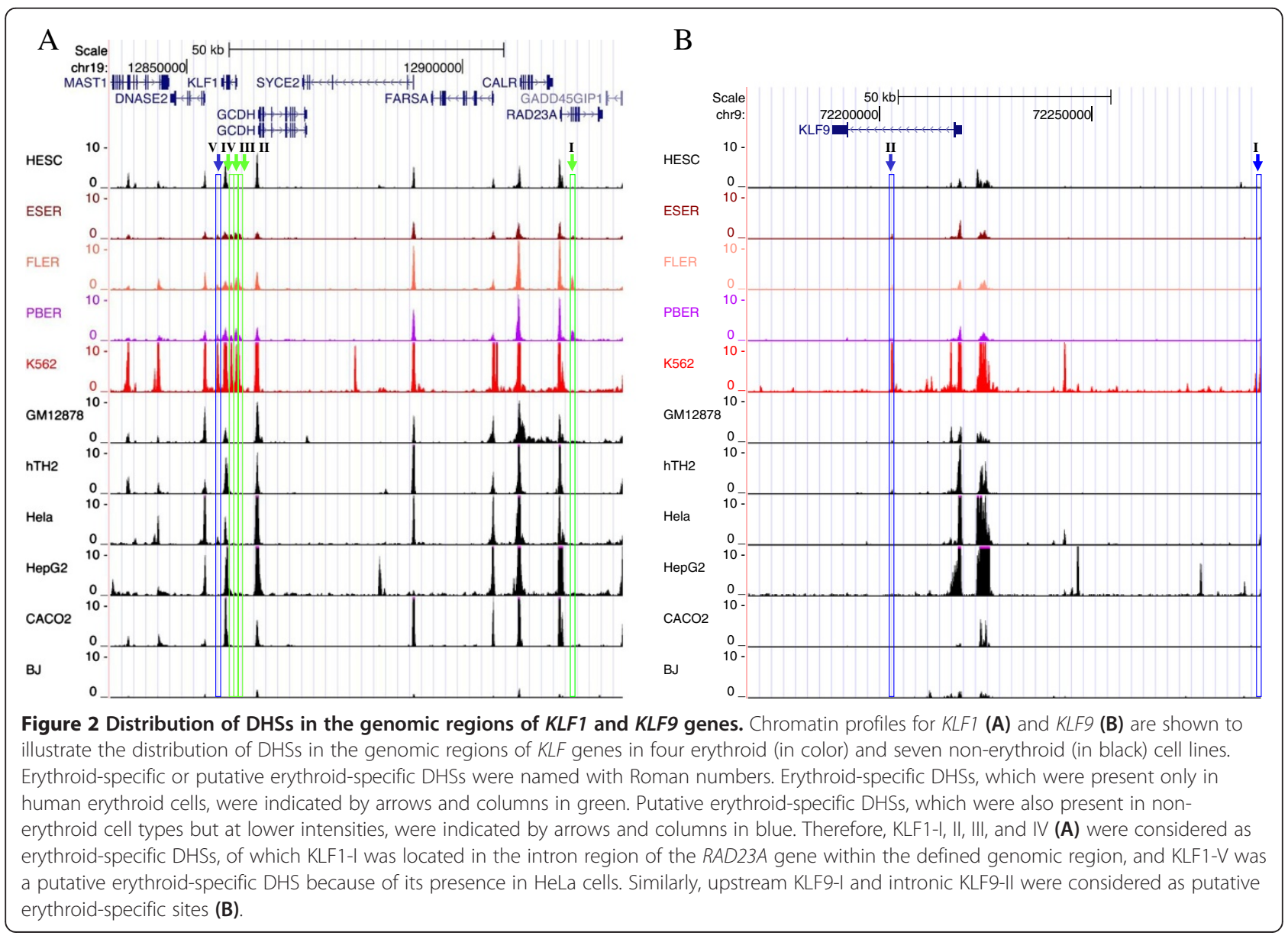


to be erythroid specific if they were only present in erythroid cells and were classified as putative erythroid specific if they were present in erythroid cells, while much subdued peaks were also detected in one or two non-erythroid cell types.

Figure 2A illustrated the DHS profiling of KLF1. Five prominent DHSs were detected in the KLF1 genomic region; of these KLF1-I was located at $>60 \mathrm{~kb}$ upstream of the KLF1 gene and was only present in three primary erythroid cells, whereas KLF1-II, III, IV, and V were located proximal to the KLF1 gene and were present in both primary erythroid cells and erythroleukemia K562 cells. KLF1-V was a putative erythroid-specific site because a small peak for this site was also present in non-erythroid HeLa cells. The diverse cellular presentation of DHSs was also observed in the profile of KLF9 (Figure 2B), with two putative erythroid-specific DHSsKLF9-I and KLF9-II-located $70 \mathrm{~kb}$ upstream or in the intron of the gene respectively.

Other erythroid-specific or putative erythroid-specific sites in the KLF profiles are shown in Additional file 1: Figure S1. Erythroid-specific DHSs include DHS-I of KLF2 (Additional file 1: Figure S1A), DHS-I and II of KLF3 (Additional file 1: Figure S1B), DHS-III and IV of KLF6 (Additional file 1: Figure S1C), DHS-I of KLF10 (Additional file 1: Figure S1D), DHS-I and II of KLF13 (Additional file 1: Figure S1F), DHS-II of KLF16 (Additional file 1: Figure S1G), and DHS-I of KLF17 (Additional file 1: Figure S1H). In addition, DHS-III of KLF3 (Additional file 1: Figure S1B), DHS-I and II of KLF6 (Additional file 1: Figure S1C), DHS-I of KLF11 (Additional file 1: Figure S1E), DHS-III of KLF13 (Additional file 1: Figure S1F), and DHS-I of KLF16 (Additional file 1: Figure S1G) were identified as putative erythroidspecific DHSs. The features of all the 23 erythroidspecific or putative erythroid-specific DHSs located in the KLF gene loci are summarized in Additional file 2: Table S1. DHS profiles of KLFs without erythroidspecific or putative erythroid-specific DHSs are shown in Additional file 1: Figure S2. It is also of interest to note that while KLF4 has been employed as a major reprogramming factor required to reverse the highly differentiated somatic cells into pluripotent cells [43], its expression in HESC was lower than that of many other KLFs, consistent with ENCODE/Caltech RNA-seq data available on UCSC Browser. As shown in Additional file 1: Figure S2A, weak peaks of DHSs (in HESC, ESER, FLER, and PBER) were found to be dispersed in the KLF4 locus, which could account for its relatively lower expression than that of the other family members in the present and previous studies [44] and its dispensable role for the self-renewal and pluripotency of ES cells $[43,45]$. In particular, DHS peaks in the KLF4 promoter region tend to decrease during erythroid differentiation, which may explain the down-regulation of KLF4 expression in erythroid cells compared with that in HESC (Figure 1).

Among the 23 prominent erythroid-specific or putative erythroid-specific DHSs, 18 (78\%) were located upstream of TSSs or downstream of poly (A) sites of the $K L F$ genes. Only four (17\%) DHSs were proximal $(<2 \mathrm{~kb})$ to TSSs, whereas $15(65 \%)$ were distal $(>10 \mathrm{~kb})$ to TSSs (Additional file 1: Figure S3A). Our data on the identified erythroid-specific DHSs are comparable to those of a previous DNase-chip report on the distribution of cell type-specific DHSs within 1\% of the human genome from six diverse cell types [3]. In contrast, with the exception of the erythroid-specific DHSs in the KLF2 and KLF17 regions (Additional file 1: Figure S1A, $H$ ), most erythroid-specific or putative erythroid-specific DHSs were present in the eight KLF genes that were up-regulated in erythroid cells (Figure 1, Figure 2, Additional file 1: Figure S1B-G). Moreover, erythroidspecific or putative erythroid-specific DHSs were absent in the genomic regions of several KLF genes (Additional file 1: Figure S2), which did not have increased expression in erythroid cells (Figure 1), implying that these KLFs may not function in erythroid cells or that they were not activated by erythroid-specific enhancers.

\section{Approximately $65 \%$ erythroid-specific or putative erythroid-specific DHSs are enhancers}

Cell type-specific DHSs have been reported to act as enhancers [3]. To identify which erythroid-specific DHSs can serve as enhancers, DLR assay was performed to evaluate the enhancer activity of 23 DHSs in driving TATA box-containing minimal promoter (minP) [46]. K562 cells are immature erythroid cells widely used in studies of erythroid differentiation or other functions of the erythroid lineage; K562 is also one of the tier 1 cell types used in the ENCODE project with massive data available for subsequent analyses. Therefore, we selected the K562 cells to identify erythroid-specific enhancers in vitro. We found that enhancer HS2 in human $\beta$ globin LCR strongly activated minP in erythroid K562 cells (Figure 3). Therefore, HS2 was chosen as the positive control for enhancer activity evaluation in this assay as previous reports did $[15,16,47]$. HS2 activated $\min P$ by approximately 5 fold in this study. Therefore, we defined DHSs that could activate minP by 5 fold, which was 2.32 after $\log _{2}$ transformation as presented in Figure 3, or higher as enhancers. We found that 15 (65\%) erythroid-specific or putative erythroid-specific DHSs had enhancer activity, with the activity of some being much stronger than that of HS2 (Figure 3), suggesting that $\min P$ was sensitive enough and sufficient for evaluating enhancer activity. Eleven (73\%) of the 15 enhancers were located in the intergenic regions and 


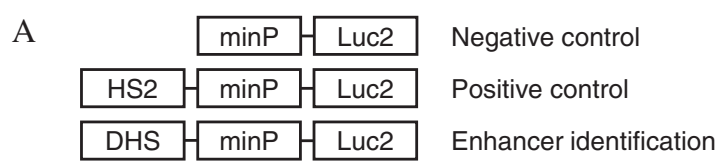

B

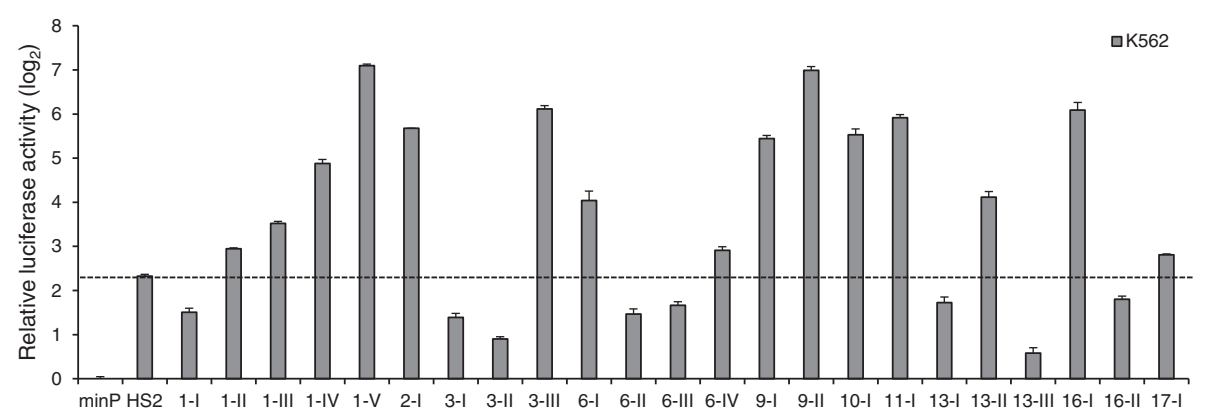

Figure 3 Enhancer identification in $\mathbf{K 5 6 2}$ cells using the DLR assay with $\mathbf{m i n P}$. (A) The constructs used in the reporter assay system are depicted. The pGL4.23 vector containing one minP was set as a negative control. The pGL4.23 construct with enhancer HS2 was set as a positive control. pGL4.23 constructs with DHSs of interest in the place of HS2 were used to evaluate the enhancer activities of DHSs. (B) Enhancer activities evaluation of 23 erythroid-specific or putative erythroid-specific DHSs in K562 cells. Each construct was transfected in triplicate at a time and transfections were repeated at least twice. For each construct, the firefly luciferase activity was normalized to that of Renilla luciferase. The relative luciferase activity was shown in $\log _{2}$ scale, with that of minP set to 0 . Standard deviations were shown as error bars above each column. DHSs with 5-fold or higher activities than that of minP were defined as enhancers.

four $(27 \%)$ were in the introns of $K L F$ genes (Additional file 1: Figure S3B). The higher frequency of enhancers in the intergenic regions is consistent with previous reports concerning enhancer distribution [3,48] and could also be explained by the fact that $83 \%$ DHSs were located beyond the proximal regions $(>2 \mathrm{~kb})$ in this study (Additional file 1: Figure S3A). The statistical analysis for enhancer distribution (Additional file 1: Figure S3B) indicated that enhancers tend to be distal [3]. Taken together, the present findings demonstrated that approximately two thirds of erythroid-specific or putative erythroid-specific DHSs were enhancers. Indeed, the majority of the DHSs were found to possess enhancer functions, supporting our original hypothesis that the high-throughput mRNA-seq and DHS mapping together provided a powerful mean for the identification of potential enhancers in the genome.

More than half of erythroid-specific or putative erythroidspecific DHSs activate KLF promoters in erythroid cells

In different cells, enhancers activate gene expression by interacting with corresponding promoters. Therefore, we evaluated the enhancer activities of all the 23 DHSs in driving their respective KLF-Ps (Additional file 2: Table S2) in K562 cells. In total, 10 KLF-Ps were cloned and their activities were examined using the DLR assay (Additional file 2: Table S3). HS2 only activated some KLF-Ps in K562, HeLa, and HEK293 cells, and thus was used as a positive reference in this assay (Figures 4 and $5)$. DHSs that significantly $(p<0.01)$ increased the activities of corresponding KLF-Ps were considered as enhancers. Of the 23 DHSs, 15 (65\%) displayed enhancer activities with respective KLF-Ps (Figure 4 and Table 1) in $K 562$ cells. Importantly, some DHSs exhibited promoter specificity; for example, DHSs such as KLF6-I, KLF10-I, KLF13-II, and KLF16-I were strong enhancers on minP, but failed to activate their own promoters. In contrast, KLF3-I, KLF6-II, KLF6-III, and KLF13-III demonstrated strong enhancer activities with KLF3, KLF6 or KLF13 promoters, but not with minP, indicating that KLF3-I, KLF6-II, KLF6-III, and KLF13-III are gene-specific enhancers, and that their enhancer activities are independent of the TATA box [49] (Table 1). The distribution of erythroid KLF enhancers is shown in Additional file 1: Figure S3C.

\section{Approximately $>67 \%$ KLF enhancers are erythroid specific}

To further investigate the erythroid specificity of KLF enhancers, we transiently transfected constructs with KLF-Ps into non-erythroid HeLa and HEK293 cells. DHSs that activated KLF-Ps in K562 cells but not in HeLa and HEK293 cells were considered as erythroidspecific KLF enhancers. DLR assay (Figure 5) revealed that 10 enhancers located in the genomic regions of KLF1-II, III, IV, and V; KLF6-II, III, and IV; KLF9-I and II; and KLF13-III were erythroid specific. Coincidentally, the mouse homologue of KLF1-II has been previously identified as an erythroid-specific enhancer [40]. These results provide strong evidence that the erythroid 


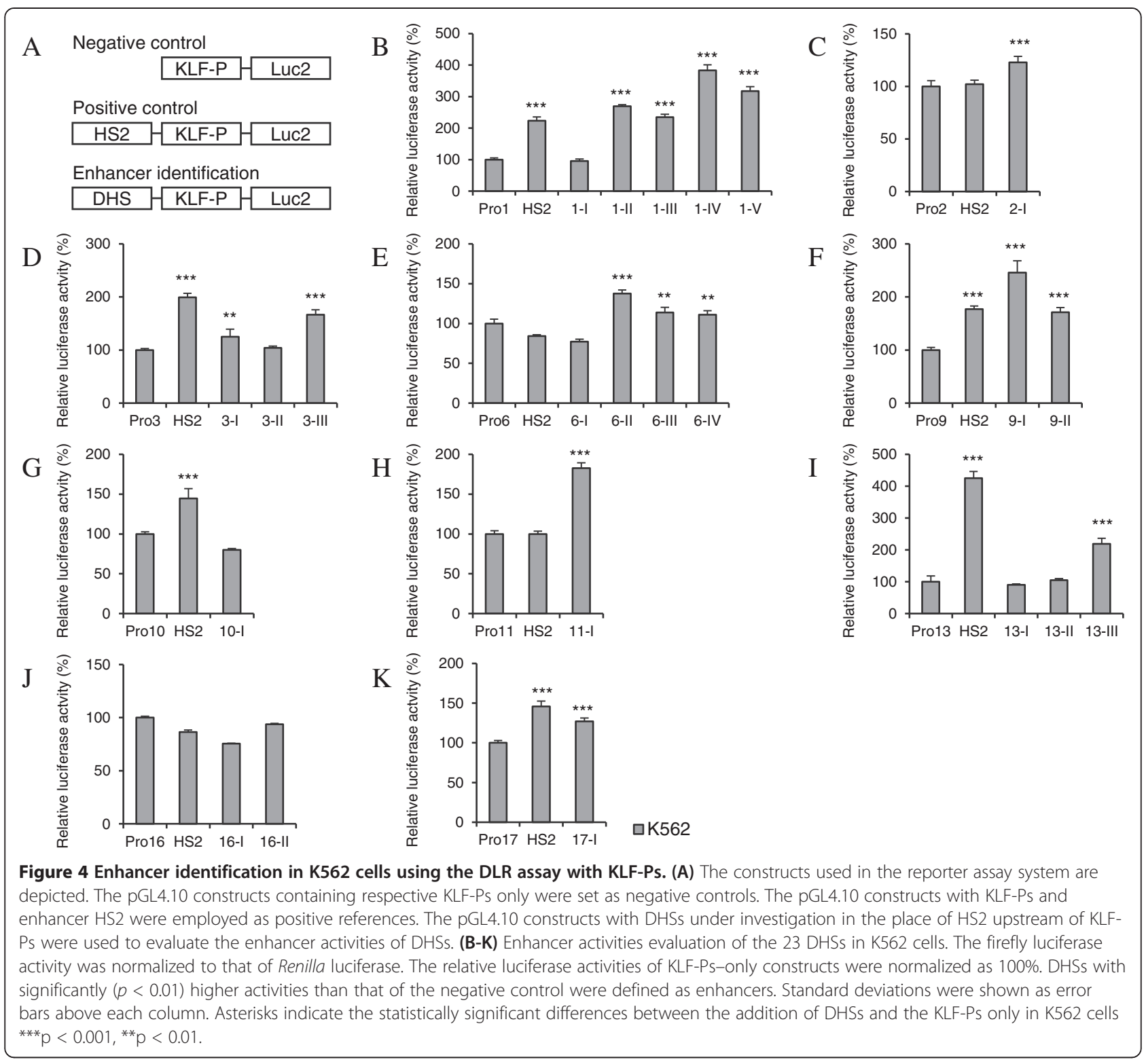

specificity of DHSs determines the erythroid specificity of enhancers. The distribution of these erythroid-specific KLF enhancers is shown in Additional file 1: Figure S3D.

\section{The nature of erythroid-specific KLF enhancers was validated by bioinformatic analyses}

In general, enhancers are characterized by species conservation [10], characteristic H3K4me1 and H3K27ac enrichment [14], chromatin accessibility [DNase I hypersensitivity, Formaldehyde-Assisted Isolation of Regulatory Elements (FAIRE) sensitivity] [2,8,50], and binding capacity of TFs and coactivators [14]. According to the UCSC Genome Browser, of the 10 erythroid-specific KLF enhancers identified in this study, only five were conserved in placental mammals [51], whereas all the
10 enhancers were enriched with H3K4me1 and/or H3K27ac modifications [52], seven of which were erythroid specific, and six were occupied by erythroid-specific TFs GATA-1 and/or NF-E2 in erythroid K562 cells [53] (Table 1), further supporting their proposed roles as erythroid-specific enhancers.

The Txn Factor ChIP track synthesizes all the available ENCODE ChIP-seq data in different cell types; these data were used to build a full view of TF-binding sites (TFBSs) on the 23 DHSs. The signal strength of TF occupancy was quantified as a cluster score ranging from 0 to 1000 . Because most (>90\%) TFBSs on the 23 DHSs occurred in K562 cells, we drew a heat map of cluster scores for this erythroid cell type after K-means clustering. As shown in Figure 6A, class II clustered four non 


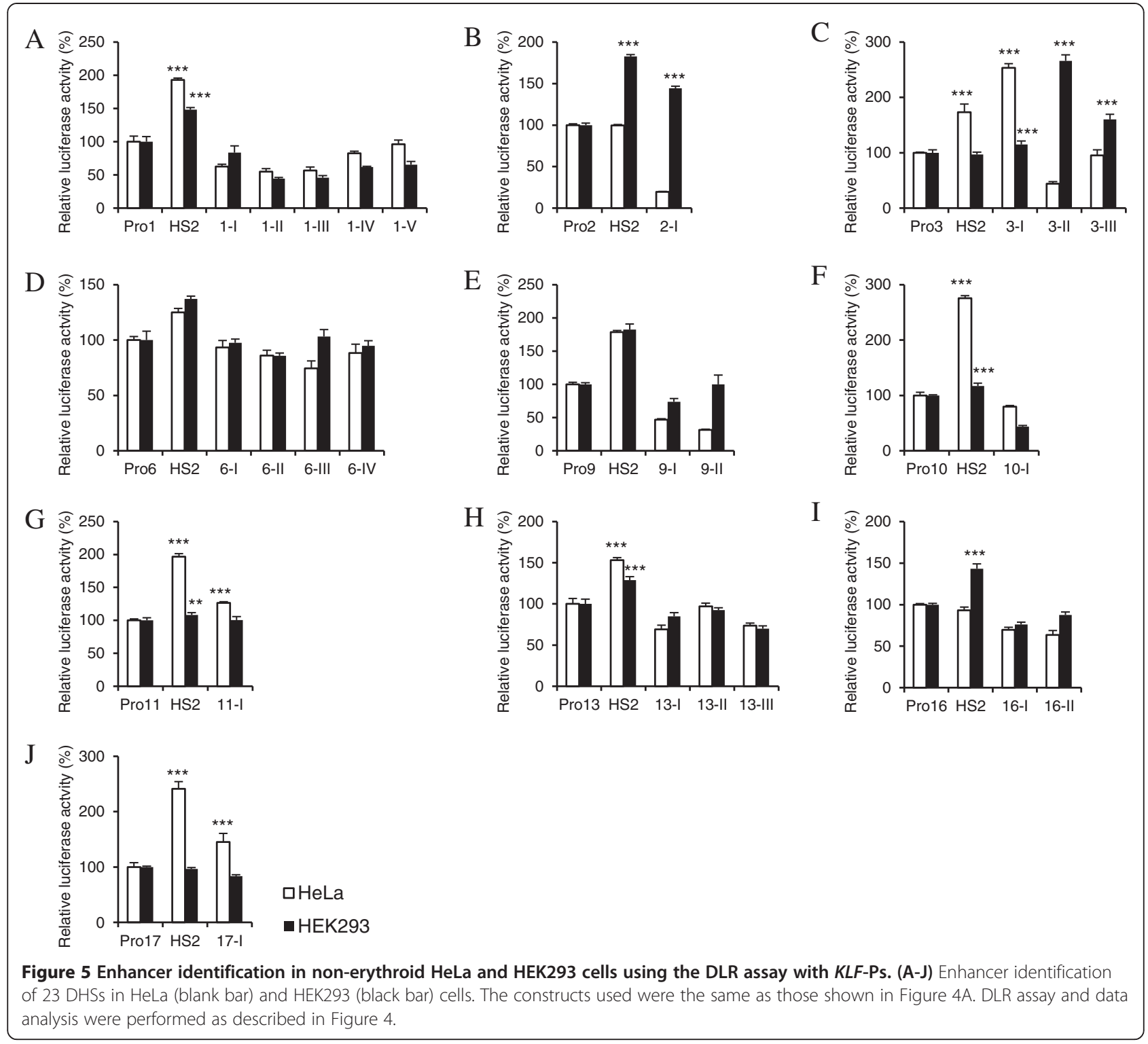

enhancers (3-II, 16-II, 1-I, and 13-I), four minP-driving enhancers (16-I, 6-I, 10-I, and 13-II), three KLF Pdriving enhancers (13-III, 6-II, and 3-I), and two minPand KLF-P-dual driving enhancers (6-IV and 3-III). These 13 DHSs were bound by fewer TFs than the 10 DHSs in class I and III, which could account for their impaired or deficient enhancer activity in $\mathrm{K} 562$ cells. Of the remaining 10 DHSs, with the exception of 6-III, all were enhancers on both promoters in $\mathrm{K} 562$ cells because of the binding of multiple TFs. Furthermore, 11-I, 17-I, and 2-I were strong enhancers in K562 cells but were not erythroid specific because of the strong binding of TFs in other cell types. The remaining seven DHSs were erythroid-specific KLF enhancers. As shown in the vertical axis, TFs clustered to different classes: class I clustered several erythroid differentiation-related TFs
(GATA-1, TAL1, and GATA-2) [22], and class II clustered enhancer-related factors (BATF, c-Fos, JunD, MEF2A, p300, PU.1, NFKB, STAT1, and SP1), histone modifiers and chromatin remodelers [HDAC2, p300, SETDB1, SIRT6, SWI/SNF components (Brg1 and Ini1), HMGN3, and Ini1] [12]. The features of TFs mentioned above were obtained from the NCBI Reference Sequence (RefSeq) database (http://www.ncbi.nlm.nih.gov/refseq/). In summary, in the open chromatin state, binding of TFs largely determines the enhancer activity of DHSs.

The ChIP-seq data of the UCSC Genome Browser did not cover all the TFBSs on the genome. Therefore, we performed de-novo motif analysis using the Multiple Em for Motif Elicitation (MEME) online software and annotated these motifs based on ENCODE-motifs by using TOMTOM [54]. ENCODE-motifs database covers 
Table 1 Characteristics of erythroid-specific or putative erythroid-specific DHSs of ten human KLF genes

\begin{tabular}{|c|c|c|c|c|c|c|c|c|c|c|c|}
\hline \multirow[t]{2}{*}{ KLF } & \multirow[t]{2}{*}{ DHS } & \multirow[t]{2}{*}{ Cs } & \multirow[t]{2}{*}{ FAIRE } & \multirow[t]{2}{*}{ H3K4me1 } & \multirow{2}{*}{$\begin{array}{l}\text { H3K27ac } \\
\text { K562 }\end{array}$} & \multirow[t]{2}{*}{ GATA-1 } & \multirow[t]{2}{*}{ NF-E2 } & \multirow{2}{*}{$\begin{array}{l}\min P \\
K 562\end{array}$} & \multicolumn{3}{|c|}{ KLF-P } \\
\hline & & & & & & & & & K562 & HeLa & HEK293 \\
\hline \multirow[t]{5}{*}{ KLF1 } & 1 & $Y$ & $Y$ & $Y$ & & & & & & & \\
\hline & $\|$ & Y & Y & $Y^{*}$ & $Y^{*}$ & Y & & Y & Y & & \\
\hline & III & Y & & $Y^{*}$ & $Y^{*}$ & Y & & Y & Y & & \\
\hline & IV & Y & Y & & $Y^{*}$ & Y & & Y & Y & & \\
\hline & V & & Y & $Y^{*}$ & $Y^{*}$ & & Y & Y & Y & & \\
\hline KLF2 & I & Y & Y & Y & Y & Y & & Y & Y & & Y \\
\hline \multirow[t]{3}{*}{ KLF3 } & I & Y & Y & Y & $Y^{*}$ & & & & Y & Y & Y \\
\hline & $\|$ & Y & Y & Y & $Y^{*}$ & & & & & & Y \\
\hline & III & Y & Y & Y & Y & & & Y & Y & & Y \\
\hline \multirow[t]{4}{*}{ KLF6 } & I & Y & Y & Y & Y & & & Y & & & \\
\hline & $\|$ & & & Y & Y & & & & Y & & \\
\hline & III & Y & & Y & $Y^{*}$ & & & & Y & & \\
\hline & IV & Y & & Y & $Y^{*}$ & & & Y & Y & & \\
\hline \multirow[t]{2}{*}{ KLF9 } & I & & Y & $Y^{*}$ & $Y^{*}$ & Y & & Y & Y & & \\
\hline & $\|$ & & Y & Y & & Y & & Y & Y & & \\
\hline KLF10 & 1 & & & $Y^{*}$ & & & & Y & & & \\
\hline KLF11 & I & Y & Y & Y & $Y^{*}$ & & & Y & Y & Y & \\
\hline \multirow[t]{3}{*}{ KLF13 } & I & & & $Y^{*}$ & & & & & & & \\
\hline & $\|$ & & & $Y^{*}$ & & & & Y & & & \\
\hline & III & & & Y & & & & & Y & & \\
\hline \multirow[t]{2}{*}{ KLF16 } & I & & Y & $Y^{*}$ & $Y^{*}$ & & & Y & & & \\
\hline & $\|$ & Y & Y & Y & Y & & & & & & \\
\hline KLF17 & I & Y & Y & $Y^{*}$ & $Y^{*}$ & & & Y & Y & Y & \\
\hline
\end{tabular}

DHS conservation (Cs) was observed across 32 placental mammals, including humans, chimps, mice, and rabbits. The chromatin accessibility using FAIRE, enhancer-associated histone modifications (H3K4me1 and H3K27ac), and TFBSs for erythroid GATA-1 and NF-E2 on DHSs in erythroid K562 cells were extracted from UCSC Genome Browser. The enhancers characterized from the erythroid-specific or putative erythroid-specific DHSs were summarized in the last four columns. Y: yes. *: this histone modification is K562 specific or putative K562 specific.

all known motifs for each factor curated from TRANSFAC, Jaspar and Protein Binding Microarray (PBM) experiments and their enrichment within corresponding TF-binding experiments, as well as novel regulatory motifs discovered by systematic application of several motif discovery tools (including MEME, MDscan, Weeder, AlignACE) and evaluated based on their enrichment relative to control motifs within TF-bound regions (Kheradpour P, Kellis M: ENCODE-motifs: systematic analysis of regulatory motifs associated with transcription factor binding in the human genome, submitted). The conserved motifs among erythroid-specific KLF enhancers are shown in Figure 6B (E-value: $5.4 \times 10^{-4}$ ) and Figure $6 \mathrm{C}$ (E-value: $2.6 \times 10^{1}$ ); the p-values of their occurrence in DHSs were $1.03 \times 10^{-11}-1.78 \times 10^{-6}$, and $4.91 \times 10^{-9}-$ $3.14 \times 10^{-5}$, respectively. Motif 1 (Figure 6B) could be the binding sites of histone modifier HDAC2 and enhancer-related TF foxa [55], and motif 2 (Figure 6C) could be the motifs of enhancer-related TFs SP1, FoxA2, PU.1 [12], and so on. The annotated motifs of
HDAC2, SP1, STAT, and PU.1 were consistent with the TFBSs in cluster II of the heat map (Figure 6A).

\section{Erythroid-specificity of KLF enhancers is validated using} the biochemical electrophoresis mobility shift assay

Because the discovered motifs were mainly enhancer related, we performed the electrophoresis mobility shift assay (EMSA) to verify the erythroid specificity of erythroid-specific KLF enhancers. KLF9-I was selected as an example. Data from the UCSC Genome Browser indicate that sequence conservation of KLF9-I was observed in neither vertebrates nor placental mammals, but it was decorated with H3K4me1 and H3K27ac enhancer marks (Table 1) and harbored binding sites of erythroid-specific GATA-1 in erythroid K562 cells (Figure 7A). Because individual TFBSs can be relatively short and degenerate, they tend to be clustered to achieve precise temporal and developmental stage specificities [56]. Factors bound to these sequences often interact with common coactivators, which, in turn, 


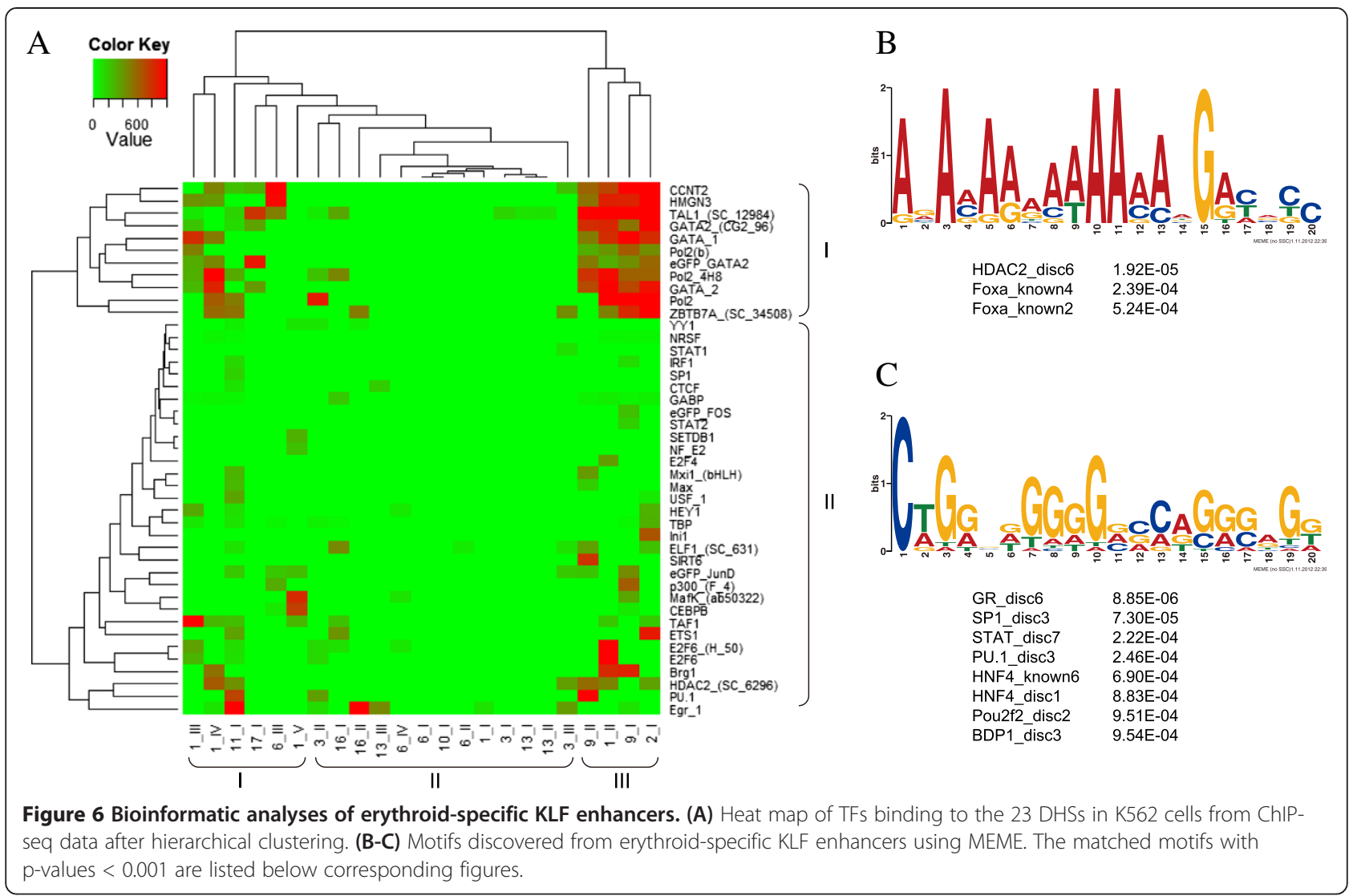

recruit the basal transcription machinery $[57,58]$. We used EMSA to further investigate whether erythroidspecific TFs or cofactor complexes bind to KLF9-I in vitro. Putative TFBSs in KLF9-I were annotated using the Txn Factor ChIP track and ENCODE-motifs on UCSC Genome Browser (Figure 7A). Sequence analysis indicated that the motif of enhancer-related protein p300 [3,14,59] was embedded in KLF9-I (Figure 7A), which may account for the enhancer nature of KLF9-I, whereas the motif of GATA-1 may account for the erythroid specificity of KLF9-I. A pair of oligos (shadowed in Figure 7A) against KLF9-I were designed using the bioinformatic analysis. The EMSA result (Figure 7B) demonstrated that at least one protein complex in erythroid K562 cell extracts (band IV) specifically bound to the oligos, whereas no such binding was observed in non-erythroid HeLa and HEK293 cell extracts, implying that an erythroid-specific TF or cofactor
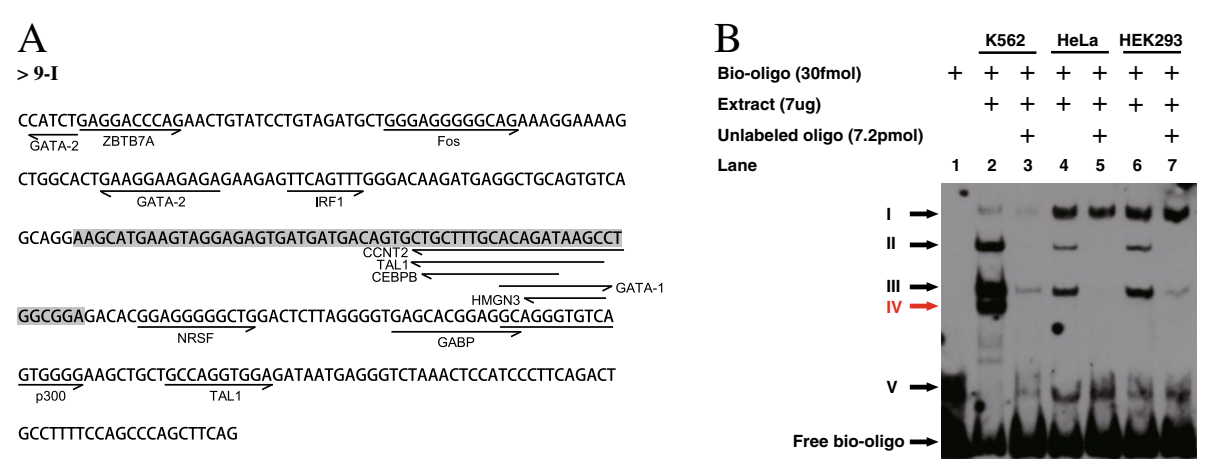

Figure 7 Biochemical analysis of erythroid-specific KLF enhancers. (A) Potential TFBSs on KLF9-I. TF occupancies on the erythroid-specific KLF enhancer KLF9-I were annotated using Txn Factor ChIP track and ENCODE-motifs on the UCSC Genome Browser. (B) Result of EMSA analysis. Biotin-labeled oligos were incubated with nuclear protein extracts of K562, HeLa, or HEK293 cells in the presence or absence of unlabeled competing oligos. Shifted bands are indicated by arrowheads on the left. Band IV (red) was found to be erythroid specific and was only detected in K562 cells, but not in HeLa or HEK293 cells. 
complex bound to KLF9-I enhancer and drives KLF9 expression in erythroid cells. The other four universal bands suggested that KLF9-I may exert some universal functions in other cell types through recruitment of some basic transcriptional regulatory protein complexes. However, KLF9 has not been shown to function in erythroid cells. The detailed mechanisms by which KLF9-I mediates transcriptional regulation of KLF9 in erythroid cells need further investigation.

\section{Discussion}

Mapping regulatory elements to the genes they regulate is of great importance to understand gene expression and functions. In this study, we provide a feasible strategy to extensively identify gene- and cell-specific enhancers from DHSs based on a rigorous and practical high-throughput sequencing technique (Figure 8). First, we refined the expression patterns of human KLFs from mRNA-seq data and proposed that the higher expression of eight KLFs in erythroid cells may be ascribed to the presence of erythroid-specific enhancers. Second, we screened erythroid-specific DHSs in the genomic regions of 17 human KLFs from DNase-seq dataset from four erythroid cell types and seven non-erythroid cell types, which largely improved the accuracy of prediction. Third, we extensively evaluated enhancer activities of all the 23 erythroid-specific or putative erythroid-specific DHSs using the DLR assay for promoter and cell-type specificities. Lastly, we validated the enhancer nature and erythroid specificity of erythroid-specific KLF enhancers through bioinformatic and biochemical analyses.

As the major contributors to cell- and developmental stage-specific gene expression, enhancers have been extensively predicted by many genome-wide approaches, including sequences conservation-, motif-, and chromatin-based computation methods [10,48,59]; ChIP-based analysis of TF binding such as CBP/p300 [13] or histone modifications (H3K4me1 and H3K27ac) [14]; or DHSs and FAIRE mapping $[3,50]$. The application of these approaches indicates that enhancers have unique properties that differentiate them from other CREs. However, enhancers predicted by these methods need to be validated further by using in vitro transient reporter gene system or in vivo transgenic systems. It is unrealistic to characterize all cell- and genespecific enhancers within the whole human genome. In addition, these methods can not simultaneously take into account both cell and gene specificities of enhancers. In this study, using the next-generation high-throughput sequencing combined with multiple enhancer activity tests, we comprehensively characterized the enhancers confined to the genomic regions of a typical family of KLFs to explore both cell and gene specificities. The expression data in mRNA-seq may indicate the presence of enhancers, and DNase-seq data suggest the location of these enhancers.

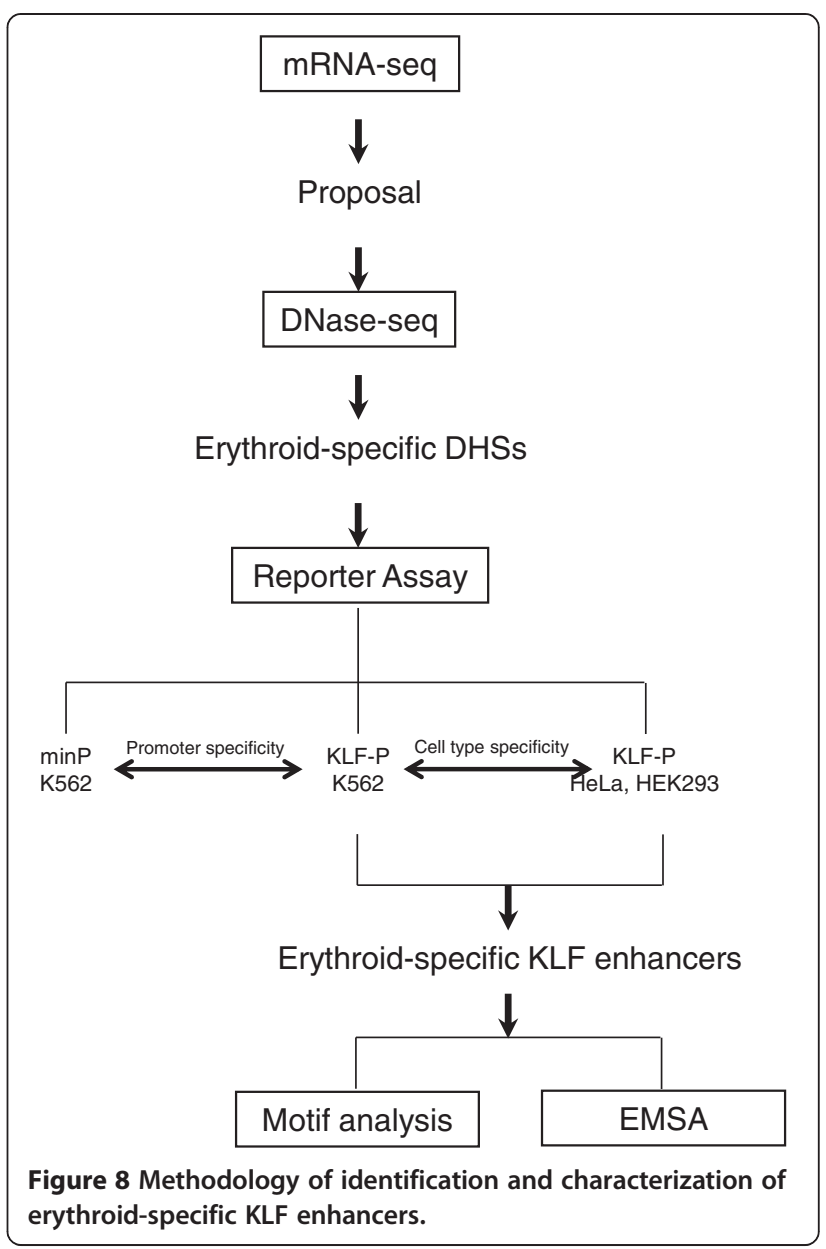

DHS mapping has higher positive rate than in-silico prediction methods and takes the advantage of unbiased property over ChIP-based methods, which only predict a subset of enhancers bound by one or several TFs and largely limited by the quality of antibodies. Reporter assays have the advantages of saving labor and time over animal models. With respect to the identification of erythroid-specific DHSs, we used cell lines originated from inner cell mass (HESC), ectoderm (HeLa), mesoderm (GM12878 and hTH2), and endoderm (HepG2 and CACO2) as control cell types for erythroid-specific DHSs screening. However, it is possible that certain erythroid-specific DHSs were excluded due to the presentation of similar DHSs as a result of binding by a different set of TFs in these non-erythroid cell lines. This setback may be remedied by the employment of highresolution genome-wide in vivo footprinting [60]. Moreover, three primary erythroid cells were used for DHSs screening, which increased the validity of erythroid-specific DHSs. Although some subtle differences, but not specificity, were observed among DHSs at different developmental stages of erythroid cells, the similarities are actually unclear (Figure 2, Additional file 1: Figures S1 and S2). K562 cells are actually suitable for identifying erythroid- 
specific enhancers. However, there appears to be a limitation in characterizing the developmental stage specificity of enhancers using this cell line because it is at an early stage of erythroid differentiation. In the future, we wish to analyze this using appropriate erythroid cell lines at different developmental stages or animal models. In reporter assays, control cell lines HEK 293 and HeLa are also representatives of different germ layers, mesoderm and ectoderm. Thus, the application of these non-erythroid control cell lines guaranteed the reliable and unbiased identification of cell-specific enhancers using our system. Beside cell-type specificity, we also explored gene specificity of enhancers, which is of great importance in gene expression and remains a challenge in this field. Furthermore, we validated the credibility of the identified erythroid-specific KLF enhancers through integrated bioinformatic and biochemical approaches to determine sequence conservation, TF binding, and histone modification markers. Identification of erythroid-specific KLF enhancers will facilitate the understanding of transcription, expression, and associated functions of $K L F$ genes in erythroid cells and provide useful information to estimate the frequency of DHSs as geneand cell-specific enhancers within the whole genome. With proper selection of cell types used as controls for DHS mapping and also employed for reporter assays, the approach described in this study should be applicable to a wide range of cells and genes of interest.

For example, a family of KLF members was selected to determine whether enhancers located in the genomic regions of KLFs were associated with their expression in erythroid cells. Here, we compared the expression of KLF mRNA transcripts in erythroid cells (ESER, FLER, and PBER) with those in non-erythroid cells (HESC) using mRNA-seq analysis. The present results reveal that eight KLFs (KLF1, KLF3, KLF6, KLF9, KLF10, KLF11, KLF13, and KLF16) show relatively higher expressions in erythroid cells than those in non-erythroid cells (Figure 1), which was proposed to be mainly determined by the presence of erythroid-specific CREs, particularly enhancers, embedded in DHSs of their genomic regions (Figure 2, Additional file 1: Figure S1, Additional file 2: Table S1). Ten erythroid-specific KLF enhancers were finally identified in the genomic regions of KLF1, KLF6, KLF9, and KLF13, indicating that these KLFs were erythroid-specific enhancers-driven genes and harbored potential biology in erythroid cells. However, despite their high expression in erythroid cells, no erythroidspecific enhancer was identified in the genomic regions of KLF3, KLF10, KLF11, and KLF16. The enhancers in the genomic regions of KLF3 and KLF11 were excluded because they demonstrated enhancer activities in nonerythroid cells as well (Figures 4 and 5, and Table 1). For KLF10 or KLF16, although the identified erythroidspecific or putative erythroid-specific DHSs in their genomic regions activated minP in $\mathrm{K} 562$ cells, no enhancer activating KLF-Ps was characterized, reflecting that DHSs identified in their genomic regions may work as promoter-specific enhancers. This is understandable because neither KLF10 nor KLF16 have been reported to be involved in erythroid differentiation and development. However, the lack of erythroid-specific enhancers in some KLFs with high erythroid expression may be caused by the limitation and bias of our arbitrary cutoffs of genomic regions. Some erythroid-specific KLF enhancers could exist beyond these confined regions (approximately $100 \mathrm{~kb}$ ) or even on different chromosomes. These missed enhancers can be found by combining our system with the genome-wide chromosome conformation capture (3C)-base technology in the future, which may help in comprehensively understanding KLF transcription in erythroid cells. DHSs in the genomic region of KLF2 and KLF17 did not appear to show erythroidspecific enhancer activity, which may account for their abolished expressions in erythroid cells during mRNAseq analysis (Figure 1A). In contrast, other KLFs (KLF4, KLF5, KLF7, KLF8, KLF12, and KLF15) were relatively highly expressed in non-erythroid cells (Figure 1), indicating that they were probably not erythroid-specific genes and that their genes expression could be driven by non-erythroid specific or universal CREs (Additional file 1: Figure S2A-E, G). No erythroid-specific or putative erythroid-specific DHSs were identified in the genomic region of KLF14 (Additional file 1: Figure S2F), which could explain why its mRNA transcript could not be detected in cell types examined in this study.

In this study we identified ten novel erythroid-specific enhancers in the genomic regions of human KLFs (KLF1, KLF6, KLF9, and KLF13), of which KLF1-II, a homolog of murine EHS1, acted as an erythroid-specific enhancer [40]. Identification of erythroid-specific KLF enhancers may reveal novel mechanisms that regulate the transcription and functions of these KLFs in erythroid cells. Till date, KLF1, KLF6, and KLF13 have been identified to be critical regulators in erythroid cells $[30,33,35,37]$. KLF9 (BTEB1) is a broadly expressed TF with high expression in the developing brain, thymus, epithelia, smooth muscle of gut and bladder, vertebrae, and cartilage primordial and is implicated to play a role in the regulation of cell proliferation and differentiation [61]. Klf9 $9^{-1-}$ mice have a normal lifespan, but impaired specific behavioral activities and decreased small intestinal villi $[62,63]$. Klf9 ${ }^{-1-}$ female mice show uterine hypoplasia, reduced litter size, increased incidence of neonatal deaths in offspring and defects in parturition [64]. At present, the biology of KLF9 in erythroid cells has not been reported; thus, the identification of erythroid-specific KLF9 enhancers implies that KLF9 could be a novel KLF member that may play critical 
roles in erythroid cells. Future studies are warranted to investigate the functions of KLF9 in hematopoiesis, and the mechanisms by which the two identified erythroidspecific KLF9 enhancers regulate KLF9 gene transcription and expression in erythroid cells.

\section{Conclusions}

The primary goal of this study is to develop a methodology to characterize enhancers from massive data generated by high-throughput sequencing technology. Using the high-throughput sequencing technique, we have provided a feasible and practical strategy to extensively identify gene- and cell-specific enhancers from DHSs. Application of our strategy led to the identification of ten erythroid-specific enhancers in the typical KLF family; their enhancer nature and erythroid specificity were confirmed using bioinformatic and biochemical analyses. Identification of erythroid-specific KLF enhancers indicates the relatively high expressions and some important functions of the corresponding KLFs in erythroid tissues.

\section{Methods}

\section{Cell culture}

K562, HeLa, and HEK293 cells were used for transient transfection to examine the enhancer activities in the DLR assay. K562 cells were cultured in RPMI1640 Medium (Gibco) with 10\% fetal bovine serum (Hyclone) and penicillin $(100 \mathrm{U} / \mathrm{ml})$-streptomycin $(0.1 \mathrm{mg} / \mathrm{ml})$ (Invitrogen), and HeLa and HEK293 cells were cultured in Dulbecco's Modified Eagle Medium (Gibco) with 10\% fetal bovine serum (Hyclone) and penicillin (100 U/ml)streptomycin $(0.1 \mathrm{mg} / \mathrm{ml})$ (Invitrogen). All cells were maintained at $37{ }^{\circ} \mathrm{C}$ with $5 \% \mathrm{CO}_{2}$ in a humidified incubator.

\section{Transcriptome sequencing and gene expression analysis} mRNA-seq was originally designed to explore the dynamic transcriptomes during human erythroid differentiation and development (Yang Y, Wang $\mathrm{H}$, Chang $\mathrm{KH}$, Qu H, Zhang Z, Xiong Q, Qi H, Cui P, Lin Q, Ruan X, et al: Transcriptome dynamics during human erythroid differentiation and development, submitted). In Brief, we extracted total RNA from HESC, ESER, FLER, and PBER, and depleted $18 \mathrm{~S}$ and $28 \mathrm{~S}$ ribosomal RNAs before constructing cDNA libraries. Next, we used the ABI SOLiD System to perform massively parallel ligation sequencing and mapped the sequence reads to human reference sequence [release Mar. 2006 (NCBI36/hg18)]. Gene expression intensity was calculated by normalizing the read counts to RPKM according to the gene length and total mapped reads, and genes with RPKM $<0.01$ were removed.

\section{Quantitative real-time PCR}

Total RNA was extracted from HESC, ESER, FLER, and PBER cells using TRIZOL ${ }^{\oplus}$ Reagent (Invitrogen, 15596018) and DNA contamination was removed using the TURBO DNA-free ${ }^{\mathrm{Tm}}$ Kit (Ambion, AM1907). DNA-free RNA was reverse transcribed using the RevertAid First Strand cDNA Synthesis Kit (Thermo Scientific, K1622) according to manufacturer's instruction. Primers were designed using Primer 5 (Additional file 2: Table S4). PCR were performed in triplicate using Maxima ${ }^{\circledR}$ SYBR Green/ROX qPCR Master Mixes $(2 \times)$ (Fermentas, K0223) and CFX96 ${ }^{\mathrm{TM}}$ Real-Time PCR Detection System (Bio-rad), and data were analyzed using the CFX Manager $^{\text {TM }}$ Software. The KLF transcript levels were first calculated by referring to those of $18 \mathrm{~S}$ ribosomal RNA, and the expression levels of KLFs in ESER, FLER, and PBER were normalized to those in HESC. The statistical significance of differences between individual KLFs' expressions in erythroid cell types and those in HESC were calculated using the independent-samples $t$-test.

\section{Digital DNase I sequencing and erythroid-specific or putative erythroid-specific DHS selection}

In this study, the DNase-seq data used were obtained from the University of Washington [41,60], and are available through the UCSC Genome Browser (http://genome.ucsc.edu) and the NCBI Gene Expression Omnibus (GEO) data repository under accessions GSE29692 and GSE32970. DHSs were identified using an algorithm developed by the University of Washington [65]. In this study, FDR threshold of $0.5 \%$ was used to define DHS for each cell type. The domains of KLF loci were defined as extensions from $70 \mathrm{~kb}$ upstream of TSSs to $20 \mathrm{~kb}$ downstream of the poly (A) sites. ESER, FLER, and PBER cells represent primary erythroid cells at different developmental stages, and K562 cells represent erythroleukemia cells. In DHS screening, all other cell types were employed as non-erythroid control cell types. DHSs in these domains were considered to be erythroid specific if they were only present in erythroid cells and were identified as putative erythroid specific if they were present in erythroid cells and exhibited much lower peaks in one or two non-erythroid cell types.

\section{DNA manipulation}

To generate firefly luciferase reporter constructs with minP, the identified 23 erythroid-specific or putative erythroid-specific DHSs were amplified from human blood genomic DNA with Pfu DNA Polymerase (Promega, M7741) and inserted upstream of minP in the pGL4.23 expression vector (Promega, E8411). To further generate firefly luciferase reporter constructs with KLF-Ps, TSSs of individual KLFs were predicted 
from UCSC Genome Browser, and fragments of approximately $1 \mathrm{~kb}$ in length upstream of TSSs (Additional file 2: Table S2) were amplified and cloned into pGL4.10 vector, followed by inserting DHSs upstream of the corresponding KLF-Ps. The activities of KLF-Ps were examined and used as baselines. HS2, a classical enhancer in $\beta$-globin LCR, was cloned into the corresponding vectors upstream of minP or KLFPs and used as positive controls in the DLR assay [16]. The primers used in this study are listed in Additional file 2: Tables S5 and S6. The integrity of the reporter constructs was confirmed using restriction digestions and sequencing.

\section{Transient transfection and DLR assay}

Cells were seeded into 48 -well plates. K562 cells $(1.5 \times$ $10^{5}$ /well) were transiently transfected with $500 \mathrm{ng}$ of firefly luciferase vector and $0.75 \mathrm{ng}$ of a Renilla luciferase vector, pRL-TK (Promega, E2441), using Lipofectamine LTX and Plus Reagent (Invitrogen, 15338-100). HeLa $\left(7 \times 10^{4} /\right.$ well $)$ and HEK293 $(7 \times$ $10^{4} /$ well) cells were similarly transfected using the Lipofectamine 2000 Transfection Reagent (Invitrogen, 11668-019) as per the manufacturer's instructions. Forty-eight hours after transfection, cells were harvested to prepare for the cell lysates, and luciferase activities were immediately measured with the Dual-Luciferase Reporter Assay System (Promega, E1910) as per the manufacturer's instructions. Transient transfections were repeated at least twice, and every construct was transfected in triplicates. Standard deviations were shown as error bars above respective columns. For data processing, firefly luciferase activity was normalized to that of Renilla luciferase in all the groups, and the relative activity of each promoter was normalized as 1 . The statistical significance of differences between promoters and DHSs were analyzed using one-way ANOVA function in $\mathrm{R}$ language.

\section{Bioinformatic analyses}

Data of conserved elements in placental mammals [51], layered H3K4me1 and layered H3K27ac [52], and Txn Factor ChIP data [53] in the regions of 23 DHSs were obtained from UCSC Genome Browser and summarized in Table 1.

TFs binding to the 23 DHSs were collected from Txn Factor ChIP track on UCSC Genome Browser. A heat map was drawn after K-means clustering using $\mathrm{R}$ language.

Conserved motifs embedded in erythroid-specific KLF enhancers were analyzed using the MEME software (http://meme.ebi.edu.au/meme/cgi-bin/meme.cgi), and these de novo discovered motifs were searched against the ENCODE-motifs database (http://www.broadinstitute. org/ pouyak/motif-disc/human/) using TOMTOM algorithm (http://meme.ebi.edu.au/meme/cgi-bin/tomtom. cgi) [54].

\section{EMSA}

Bio-11-dUTP (Ambion, AM8450) and TdT (New England Biolabs, M0315s) were used to label the $3^{\prime}-\mathrm{OH}$ of singlestranded oligos (5' -AGC ATG AAG TAG GAG AGT GAT GAT GAC AGT GCT GCT TTG CAC AGA TAA GCC TGG CGG A-3', 5'-TCC GCC AGG CTT ATC TGT GCA AAG CAG CAC TGT CAT CAT CAC TCT CCT ACT TCA TGC T-3') and complementary oligos were annealed as per the manufacturer's instructions. Nuclear proteins of K562, HeLa, and HEK293 cells were extracted using the rapid micro-preparation method (lysis buffer: $10 \mathrm{mM}$ Hepes [pH7.9], $10 \mathrm{mM} \mathrm{KCl}, 1.5 \mathrm{mM} \mathrm{MgCl}_{2}$, $0.5 \mathrm{mM}$ PMSF, $0.5 \mathrm{mM}$ DTT; high-salt extraction buffer: $20 \mathrm{mM}$ Hepes [pH7.9], 25\% glycerol, $0.42 \mathrm{M} \mathrm{NaCl}, 1.5 \mathrm{mM}$ $\mathrm{MgCl}_{2}, 0.2 \mathrm{mM}$ EDTA, 0.5 mM PMSF, 0.5 mM DTT) [66]. Protein concentrations were measured using the BCA Protein Assay Kit (Pierce, 23225). EMSA was performed in $20 \mu$ reaction mixture, containing 30 fmol biotin-labeled oligos and $7 \mu \mathrm{g}$ nuclear extract with or without $7.2 \mathrm{pmol}$ unlabeled oligos using the LightShift Chemiluminescent EMSA Kit (Pierce, 20148) according to the manufacturer's instruction (Figure 7B).

\section{Availability of supporting data}

DNase-seq data are available through the UCSC Genome Browser (http://genome.ucsc.edu/cgi-bin/hgFileUi? $\mathrm{db}=$ hg19\&g=wgEncodeUwDnase), or through the NCBI Gene Expression Omnibus (GEO) data repository (accession numbers: GSE29692, GSE32970). The other data sets supporting the results of this article are included within the article and the additional files.

\section{Additional files}

Additional file 1: Figure S1. Chromatin profiles of KLF genes containing erythroid-specific (arrow and column in green) or putative erythroid-specific (arrow and column in blue) DHSs. KLF loci were arbitrarily defined as extension from $70 \mathrm{~kb}$ upstream of the TSSs to $20 \mathrm{~kb}$ downstream of the poly (A) sites. Erythroid-specific or putative erythroidspecific DHSs were respectively marked with green and blue arrowheads and named with Roman numbers. Figure S2. Chromatin profiles of KLF genes without erythroid-specific or putative erythroid-specific DHSs. Figure S3. Distribution statistics of the identified erythroid-specific or putative erythroid-specific DHSs and enhancers in the genomic regions of KLFs. A. Statistics of the distribution of identified erythroid-specific or putative erythroid-specific DHSs relative to KLF genes and TSSs. In total, $18(78 \%)$ and five (22\%) DHSs are localized to the intergenic and intronic regions, respectively; 15 (65.2\%) DHSs are located far distal (>10 kb) to TSSs, four (17.4\%) DHSs are located distal (2-10 kb) to TSSs, and four $(17.4 \%)$ DHSs are located in proximal $(<2 \mathrm{~kb})$ promoter regions. DHS KLF1-III contains TSS (Additional file 1: Table S1). B. Statistics of the distribution of the identified enhancers under the control of minP in K562 cells relative to KLF genes and TSSs. C. Statistics of the identified erythroid KLF enhancer distribution relative to the KLF genes and TSSs. 
D. Statistics of the identified erythroid-specific KLF enhancer distribution relative to KLF genes and TSSS.

Additional file 2: Table S1. The relative position and length of 23 identified erythroid-specific or putative erythroid-specific DHSs. Table S2. Position, length, GC content, chromatin accessibility (DNase I hypersensitivity), and references of ten KLF promoters used in enhancer assays. Table S3. Activities of KLF promoters measured by the luciferase reporter assay. Table S4. Primers used for measuring the expression patterns of KLFs in real-time PCR. Table S5. Primers used for amplification of erythroidspecific DHS fragments inserted in luciferase reporter constructs. Table S6. Primers used for amplification of KLF promoters. Supplementary References.

\section{Abbreviations}

DHS: DNase I hypersensitive site; CRE: Cis-regulatory element; KLF: Krüppellike factor; DLR: Dual-luciferase reporter; minP: Minimal promoter; KLF-P: KLF promoter; ChIP: Chromatin immunoprecipitation; LCR: Locus control region; ENCODE: ENCyclopedia of DNA Elements; TF: Transcription factor: HESC: Human embryonic stem cells; ESER: Embryonic stem cells-derived erythroid cells; FLER: Fetal liver-derived erythroid cells; PBER: Adult mobilized peripheral blood CD34+ cells derived erythroid cells; FDR: False discovery rate; TSS: Transcription start site; FAIRE: Formaldehyde-assisted isolation of regulatory elements; TFBS: TF binding site; MEME: Multiple Em for Motif Elicitation; EMSA: Electrophoresis mobility shift assay; RPKM: Reads per kilobase of exon model per million mapped reads.

\section{Competing interests}

The authors declare no competing interests.

\section{Authors' contributions}

QX and ZZ carried out molecular genetic studies, DLR assay, EMSA and wrote the paper; KHC performed cell culture and wrote the paper; $Y L$ and $X R$ performed cell culture; HQ, RS, PJS and JAS performed high-throughput DNase I profiling and analyzed data; HW and YY performed high-throughput transcriptome sequencing; $Y Y$ analyzed mRNA-seq data and performed the bioinformatic and statistical analyses; $H Q$ and YL performed RT-PCR and DLR assay; GS and QL helped to draft the manuscript; JAS and XF conceived of the study, and participated in its design and coordination and wrote the paper. All authors read and approved the final manuscript.

\section{Acknowledgements}

The authors thank Dr. Yuxia Jiao for critically reading the manuscript. This research was supported by the "Strategic Priority Research Program" of the Chinese Academy of Sciences, Stem Cell and Regenerative Medicine Research (XDA01040405 to XF); National Natural Science Foundation of China (30971673 to XF and 31100924 to YL); the "863 Projects" of Ministry of Science and Technology of China (2011AA020118 to XF); the National Key Scientific Instrument and Equipment Development Projects of China (2011YQ03013404 to XF); and National Institute of Health grants of United States (1RC2HG005654 to JAS).

\section{Author details}

'CAS Key Laboratory of Genome Sciences and Information, Beijing Institute of Genomics, Chinese Academy of Sciences, Beijing 100101, P.R. China. ${ }^{2}$ University of Chinese Academy of Sciences, Beijing 100049, P.R. China. ${ }^{3}$ Division of Hematology, Department of Medicine, University of Washington, Seattle, WA 98195, USA. ${ }^{4}$ Department of Genome Sciences, University of Washington, Seattle, WA 98195, USA. ${ }^{5}$ Division of Medical Genetics, Department of Medicine, University of Washington, Seattle, WA 98195, USA.

Received: 18 March 2013 Accepted: 23 August 2013

Published: 28 August 2013

\section{References}

1. Maston GA, Evans SK, Green MR: Transcriptional regulatory elements in the human genome. Annu Rev Genomics Hum Genet 2006, 7:29-59.

2. Boyle AP, Davis S, Shulha HP, Meltzer P, Margulies EH, Weng Z, Furey TS, Crawford GE: High-resolution mapping and characterization of open chromatin across the genome. Cell 2008, 132(2):311-322.

3. Xi H, Shulha HP, Lin JM, Vales TR, Fu Y, Bodine DM, McKay RD, Chenoweth JG, Tesar PJ, Furey TS, et al: Identification and characterization of cell type-specific and ubiquitous chromatin regulatory structures in the human genome. PLoS Genet 2007, 3(8):e136.

4. Birney E, Stamatoyannopoulos JA, Dutta A, Guigo R, Gingeras TR, Margulies EH, Weng Z, Snyder M, Dermitzakis ET, Thurman RE, et al: Identification and analysis of functional elements in $1 \%$ of the human genome by the ENCODE pilot project. Nature 2007, 447(7146):799-816.

5. Gross DS, Garrard WT: Nuclease hypersensitive sites in chromatin. Annu Rev Biochem 1988, 57:159-197.

6. Wu C: The $5^{\prime}$ ends of Drosophila heat shock genes in chromatin are hypersensitive to DNase I. Nature 1980, 286(5776):854-860.

7. Crawford GE, Davis S, Scacheri PC, Renaud G, Halawi MJ, Erdos MR, Green R, Meltzer PS, Wolfsberg TG, Collins FS: DNase-chip: a high-resolution method to identify DNase I hypersensitive sites using tiled microarrays. Nat Methods 2006, 3(7):503-509.

8. Song L, Zhang Z, Grasfeder LL, Boyle AP, Giresi PG, Lee BK, Sheffield NC, Graf S, Huss M, Keefe D, et al: Open chromatin defined by DNasel and FAIRE identifies regulatory elements that shape cell-type identity. Genome Res 2011, 21(10):1757-1767

9. Shu W, Chen H, Bo X, Wang S: Genome-wide analysis of the relationships between DNasel HS, histone modifications and gene expression reveals distinct modes of chromatin domains. Nucleic Acids Res 2011, 39(17):7428-7443.

10. Visel A, Prabhakar S, Akiyama JA, Shoukry M, Lewis KD, Holt A, Plajzer-Frick I, Afzal V, Rubin EM, Pennacchio LA: Ultraconservation identifies a small subset of extremely constrained developmental enhancers. Nat Genet 2008, 40(2):158-160.

11. Banerji J, Rusconi S, Schaffner W: Expression of a beta-globin gene is enhanced by remote SV40 DNA sequences. Cell 1981, 27(2 Pt 1):299-308.

12. Jin F, Li Y, Ren B, Natarajan R: Enhancers: multi-dimensional signal integrators. Transcription 2011, 2(5):226-230.

13. Visel A, Blow MJ, Li Z, Zhang T, Akiyama JA, Holt A, Plajzer-Frick I, Shoukry $M$, Wright $C$, Chen F, et al: ChIP-seq accurately predicts tissue-specific activity of enhancers. Nature 2009, 457(7231):854-858.

14. Heintzman ND, Hon GC, Hawkins RD, Kheradpour P, Stark A, Harp LF, Ye Z, Lee LK, Stuart RK, Ching CW, et al: Histone modifications at human enhancers reflect global cell-type-specific gene expression. Nature 2009, 459(7243):108-112

15. Li Q, Peterson KR, Fang X, Stamatoyannopoulos G: Locus control regions. Blood 2002, 100(9):3077-3086.

16. Tuan DY, Solomon WB, London IM, Lee DP: An erythroid-specific, developmental-stage-independent enhancer far upstream of the human "beta-like globin" genes. Proc Natl Acad Sci U S A 1989, 86(8):2554-2558.

17. Suzuki M, Moriguchi T, Ohneda K, Yamamoto M: Differential contribution of the Gata1 gene hematopoietic enhancer to erythroid differentiation. Mol Cell Biol 2009, 29(5):1163-1175.

18. Delabesse E, Ogilvy S, Chapman MA, Piltz SG, Gottgens B, Green AR: Transcriptional regulation of the SCL locus: identification of an enhancer that targets the primitive erythroid lineage in vivo. Mol Cell Biol 2005, 25(12):5215-5225.

19. Lacronique V, Lopez S, Miquerol L, Porteu A, Kahn A, Raymondjean M: Identification and functional characterization of an erythroid-specific enhancer in the L-type pyruvate kinase gene. J Biol Chem 1995, 270(25):14989-14997.

20. Surinya KH, Cox TC, May BK: Identification and characterization of a conserved erythroid-specific enhancer located in intron 8 of the human 5 aminolevulinate synthase 2 gene. J Biol Chem 1998, 273(27):16798-16809.

21. Pearson R, Fleetwood J, Eaton S, Crossley M, Bao S: Krüppel-like transcription factors: a functional family. Int J Biochem Cell Biol 2008, 40(10):1996-2001.

22. Schechter AN: Hemoglobin research and the origins of molecular medicine. Blood 2008, 112(10):3927-3938.

23. Miller IJ, Bieker JJ: A novel, erythroid cell-specific murine transcription factor that binds to the CACCC element and is related to the Krüppel family of nuclear proteins. Mol Cell Biol 1993, 13(5):2776-2786.

24. Zhou D, Liu K, Sun CW, Pawlik KM, Townes TM: KLF1 Regulates BCL11A expression and gamma- to beta-globin gene switching. Nat Genet 2010, 42(9):742-744.

25. Marini MG, Porcu L, Asunis I, Loi MG, Ristaldi MS, Porcu S, Ikuta T, Cao A, Moi P: Regulation of the human HBA genes by KLF4 in erythroid cell lines. Br J Haematol 2010, 149(5):748-758.

26. Kalra IS, Alam MM, Choudhary PK, Pace BS: Krüppel-like factor 4 activates HBG gene expression in primary erythroid cells. Br J Haematol 2011, 154(2):248-259. 
27. Basu P, Morris PE, Haar JL, Wani MA, Lingrel JB, Gaensler KM, Lloyd JA: KLF2 Is essential for primitive erythropoiesis and regulates the human and murine embryonic beta-like globin genes in vivo. Blood 2005, 106(7):2566-2571.

28. Asano H, Li XS, Stamatoyannopoulos G: FKLF, a novel Krüppel-like factor that activates human embryonic and fetal beta-like globin genes. Mol Cell Biol 1999, 19(5):3571-3579.

29. Emery DW, Gavriilidis G, Asano H, Stamatoyannopoulos G: The transcription factor KLF11 can induce gamma-globin gene expression in the setting of in vivo adult erythropoiesis. J Cell Biochem 2007, 100(4):1045-1055.

30. Asano H, Li XS, Stamatoyannopoulos G: FKLF-2: a novel Krüppel-like transcriptional factor that activates globin and other erythroid lineage genes. Blood 2000, 95(11):3578-3584.

31. Ma XY, Wang MJ, Qu XH, Xing GC, Zhu YP, He FC: Transcriptional regulation of gamma- and epsilon-globin genes by basic Krüppel-like factor. Sheng Wu Hua Xue Yu Sheng Wu Wu Li Xue Bao (Shanghai) 2003, 35(3):271-276.

32. Zhang P, Basu P, Redmond LC, Morris PE, Rupon JW, Ginder GD, Lloyd JA: A functional screen for Krüppel-like factors that regulate the human gamma-globin gene through the CACCC promoter element. Blood Cells Mol Dis 2005, 35(2):227-235.

33. Matsumoto N, Kubo A, Liu H, Akita K, Laub F, Ramirez F, Keller G, Friedman SL: Developmental regulation of yolk sac hematopoiesis by Kruppel-like factor 6. Blood 2006, 107(4):1357-1365.

34. Huber TL, Perkins AC, Deconinck AE, Chan FY, Mead PE, Zon LI: Neptune, a Krüppel-like transcription factor that participates in primitive erythropoiesis in Xenopus. Curr Biol 2001, 11(18):1456-1461.

35. Perkins $A C$, Sharpe $A H$, Orkin $S H$ : Lethal beta-thalassaemia in mice lacking the erythroid CACCC-transcription factor EKLF. Nature 1995, 375(6529):318-322.

36. Funnell AP, Norton LJ, Mak KS, Burdach J, Artuz CM, Twine NA, Wilkins MR, Power CA, Hung TT, Perdomo J, et al: The CACCC-binding protein KLF3/ BKLF represses a subset of KLF1/EKLF target genes and is required for proper erythroid maturation in vivo. Mol Cell Biol 2012, 32(16):3281-3292.

37. Gordon AR, Outram SV, Keramatipour M, Goddard CA, Colledge WH, Metcalfe JC, Hager-Theodorides AL, Crompton T, Kemp PR: Splenomegaly and modified erythropoiesis in KLF13-/- mice. J Biol Chem 2008, 283(18):11897-11904.

38. Basu P, Lung TK, Lemsaddek W, Sargent TG, Williams DC Jr, Basu M, Redmond LC, Lingrel JB, Haar JL, Lloyd JA: EKLF and KLF2 have compensatory roles in embryonic beta-globin gene expression and primitive erythropoiesis. Blood 2007, 110(9):3417-3425.

39. Funnell AP, Maloney CA, Thompson $\sqcup$, Keys J, Tallack M, Perkins AC, Crossley M: Erythroid Krüppel-like factor directly activates the basic Krüppel-like factor gene in erythroid cells. Mol Cell Biol 2007, 27(7):2777-2790

40. Chen X, Reitman M, Bieker JJ: Chromatin structure and transcriptional control elements of the erythroid Krüppel-like factor (EKLF) gene. J Biol Chem 1998, 273(39):25031-25040

41. Thurman RE, Rynes E, Humbert R, Vierstra J, Maurano MT, Haugen E, Sheffield NC, Stergachis $A B$, Wang $H$, Vernot $B$, et al: The accessible chromatin landscape of the human genome. Nature 2012, 489(7414):75-82

42. Phillips JE, Corces VG: CTCF: master weaver of the genome. Cell 2009, 137(7):1194-1211.

43. Takahashi K, Yamanaka S: Induction of pluripotent stem cells from mouse embryonic and adult fibroblast cultures by defined factors. Cell 2006, 126(4):663-676.

44. Segre JA, Bauer C, Fuchs E: KIf4 is a transcription factor required for establishing the barrier function of the skin. Nat Genet 1999, 22(4):356-360

45. Jiang J, Chan YS, Loh YH, Cai J, Tong GQ, Lim CA, Robson P, Zhong S, Ng $\mathrm{HH}$ : A core Klf circuitry regulates self-renewal of embryonic stem cells. Nat Cell Biol 2008, 10(3):353-360.

46. Lee EY, Ji H, Ouyang Z, Zhou B, Ma W, Vokes SA, McMahon AP, Wong WH, Scott MP: Hedgehog pathway-regulated gene networks in cerebellum development and tumorigenesis. Proc Natl Acad Sci 2010, 107(21):9736.

47. Petrykowska HM, Vockley CM, Elnitski L: Detection and characterization of silencers and enhancer-blockers in the greater CFTR locus. Genome Res 2008, 18(8):1238-1246.

48. Hallikas O, Palin K, Sinjushina N, Rautiainen R, Partanen J, Ukkonen E, Taipale $\mathrm{J}$ : Genome-wide prediction of mammalian enhancers based on analysis of transcription-factor binding affinity. Cell 2006, 124(1):47-59.

49. Gehrau RC, D'Astolfo DS, Prieto C, Bocco JL, Koritschoner NP: Genomic organization and functional analysis of the gene encoding the Krüppellike transcription factor KLF6. Biochim Biophys Acta 2005, 1730(2):137-146.
50. Giresi PG, Kim J, McDaniell RM, lyer VR, Lieb JD: FAIRE (formaldehydeassisted isolation of regulatory elements) isolates active regulatory elements from human chromatin. Genome Res 2007, 17(6):877-885.

51. Siepel A, Bejerano G, Pedersen JS, Hinrichs AS, Hou M, Rosenbloom K, Clawson H, Spieth J, Hillier LW, Richards S, et al: Evolutionarily conserved elements in vertebrate, insect, worm, and yeast genomes. Genome Res 2005, 15(8):1034-1050.

52. Mikkelsen TS, Ku M, Jaffe DB, Issac B, Lieberman E, Giannoukos G, Alvarez P, Brockman W, Kim TK, Koche RP, et al: Genome-wide maps of chromatin state in pluripotent and lineage-committed cells. Nature 2007, 448(7153):553-560.

53. Euskirchen GM, Rozowsky JS, Wei CL, Lee WH, Zhang ZD, Hartman S, Emanuelsson O, Stolc V, Weissman S, Gerstein MB, et al: Mapping of transcription factor binding regions in mammalian cells by ChIP: comparison of array- and sequencing-based technologies. Genome Res 2007, 17(6):898-909.

54. Bailey TL, Boden M, Buske FA, Frith M, Grant CE, Clementi L, Ren J, Li WW, Noble WS: MEME SUITE: tools for motif discovery and searching. Nucleic Acids Res 2009, 37(Web Server issue):W202-W208.

55. Lupien M, Eeckhoute J, Meyer CA, Wang Q, Zhang Y, Li W, Carroll JS, Liu XS, Brown M: FoxA1 Translates epigenetic signatures into enhancer-driven lineage-specific transcription. Cell 2008, 132(6):958-970.

56. Kadonaga JT: Regulation of RNA polymerase II transcription by sequencespecific DNA binding factors. Cell 2004, 116(2):247-257.

57. Blackwood EM, Kadonaga JT: Going the distance: a current view of enhancer action. Science 1998, 281(5373):60-63.

58. Carter D, Chakalova L, Osborne CS, Dai YF, Fraser P: Long-range chromatin regulatory interactions in vivo. Nat Genet 2002, 32(4):623-626.

59. Heintzman ND, Stuart RK, Hon G, Fu Y, Ching CW, Hawkins RD, Barrera LO, Van Calcar S, Qu C, Ching KA, et al: Distinct and predictive chromatin signatures of transcriptional promoters and enhancers in the human genome. Nat Genet 2007, 39(3):311-318

60. Hesselberth JR, Chen X, Zhang Z, Sabo PJ, Sandstrom R, Reynolds AP, Thurman RE, Neph S, Kuehn MS, Noble WS, et al: Global mapping of protein-DNA interactions in vivo by digital genomic footprinting. Nat Methods 2009, 6(4):283-289.

61. Martin KM, Metcalfe JC, Kemp PR: Expression of Klf9 and Klf13 in mouse development. Mech Dev 2001, 103(1-2):149-151.

62. Morita M, Kobayashi A, Yamashita T, Shimanuki T, Nakajima O, Takahashi S, Ikegami S, Inokuchi K, Yamashita K, Yamamoto M, et al: Functional analysis of basic transcription element binding protein by gene targeting technology. Mol Cell Biol 2003, 23(7):2489-2500.

63. Simmen FA, Xiao R, Velarde MC, Nicholson RD, Bowman MT, Fujii-Kuriyama Y, Oh SP, Simmen RC: Dysregulation of intestinal crypt cell proliferation and villus cell migration in mice lacking Kruppel-like factor 9. Am J Physiol Gastrointest Liver Physiol 2007, 292(6):G1757-1769.

64. Simmen RC, Eason RR, McQuown JR, Linz AL, Kang TJ, Chatman L Jr, Till SR, Fujii-Kuriyama Y, Simmen FA, Oh SP: Subfertility, uterine hypoplasia, and partial progesterone resistance in mice lacking the Kruppel-like factor 9/ basic transcription element-binding protein-1 (Bteb1) gene. J Biol Chem 2004, 279(28):29286-29294.

65. John S, Sabo PJ, Thurman RE, Sung MH, Biddie SC, Johnson TA, Hager GL, Stamatoyannopoulos JA: Chromatin accessibility pre-determines glucocorticoid receptor binding patterns. Nat Genet 2011, 43(3):264-268.

66. Andrews NC, Faller DV: A rapid micropreparation technique for extraction of DNA-binding proteins from limiting numbers of mammalian cells. Nucleic Acids Res 1991, 19(9):2499.

\section{doi:10.1186/1471-2164-14-587}

Cite this article as: Xiong et al:: Comprehensive characterization of erythroid-specific enhancers in the genomic regions of human Krüppellike factors. BMC Genomics 2013 14:587. 